\title{
Primary Adenocarcinoma of middle ear with intracranial and parotid extension - A rare case report and literature review.
}

\begin{tabular}{|c|c|}
\hline \multicolumn{2}{|c|}{ Volume : 6 | Issue : 02 | December 2018• ISSN No 2321-3450 | Page: 27 - 32} \\
\hline Date Received: 15.11 .2018 & Date Published: 28.11 .2018 \\
\hline \multicolumn{2}{|c|}{ Review Process: Double blind Peer Reviewed by Editorial Board Members } \\
\hline \multicolumn{2}{|l|}{ Authors: } \\
\hline \multicolumn{2}{|c|}{$\begin{array}{l}\text { Gupta Chhavi (1), Sharma Sumit (2), Bhargava P (3) } \\
\text { Assistant Professor(1) Associate Professor(2), Department of ENT, Mayo Institute of Medical } \\
\text { Sciences, Gadia, Barabanki, Professor(3), Department of Oto-rhinolaryngology, R. D. Gardi } \\
\text { Medical College, Ujjain, MP }\end{array}$} \\
\hline \multicolumn{2}{|c|}{$\begin{array}{l}\text { Corresponding author: } \\
\text { Dr. Sharma Sumit Associate Professor, Department of Otorhinolaryngology, } \\
\text { Mayo Institute of Medical Scienc4es,. Gadia, Barabanki. }\end{array}$} \\
\hline
\end{tabular}

\section{ABSTRACT}

Adenocarcinoma of middle ear is a rare tumor; few cases were reported in literature. Primary middle ear Adenocarcinoma is locally invasive neoplasm arises from middle ear mucosa. These tumors may or may not be associated with Chronic Suppurative Otitis media. These tumors tend to have slow growth pattern and have an infrequent incidence of distant metastasis, recurrence is also a common problem. . They should be considered in the differential diagnosis of lytic lesions of the temporal bone on imaging. We reported a histologically proven case of Adenocarcinoma of middle ear with intracranial and parotid extension in $24 \mathrm{yr}$ old female patient that was successfully managed. If managed early they have a very god prognosis with a 5 -year survival of $70 \%$ has been reported for low-grade Adenocarcinoma.

Keywords: Adenocarcinoma, middle ear, invasive neoplasm 


\section{CASE REPORT}

Primary Adenocarcinoma of middle ear with intracranial and parotid extension A rare case and literature review.

\section{INTRODUCTION}

Primary Middle ear Adenocarcinoma is a very rare, locally invasive neoplasm assumed to arise from the middle ear mucosa. The tumour may or may not be associated with Chronic Suppurative Otitis media. Some authors have concluded that middle ear Adenocarcinoma belongs to the spectrum of extra cranial tumors that may have possible local extension to the brain.

Although endolymphatic sac tumor (aggressive papillary middle ear tumor) and jugulotympanic paraganglioma may show brain invasion, intracranial extension of histologically confirmed middle ear adenocarcinoma has not been previously reported.

Adenocarcinomas must be differentiated from benign adenomas. Adenocarcinomas are best managed by aggressive surgical resection, with postoperative irradiation used in cases of incomplete resection or high-grade tumors.

The findings prior to histologic examination were unusual in case of adenocarcinoma as they were consistent with a tumor of the glomus tympanicum or glomus jugulare.

\section{CASE REPORT:}

A 24yr old female patient presented with chief complaint of

1. Discharging right ear since $2 \mathrm{yr}$ which was initially intermittent, moderate in amount, whitish in colour, non foul smelling, not blood stained patient took treatment for this in private clinic and relieved for $1 \mathrm{yr}$ but she again c/o discharge after 1 yr which was continuous, yellow colored, foul smelling, blood stained.

2. She also c/o pain in right ear since 2 years initially it was intermittent, mild, localized, not radiating but since 2 months it was severe in nature. 


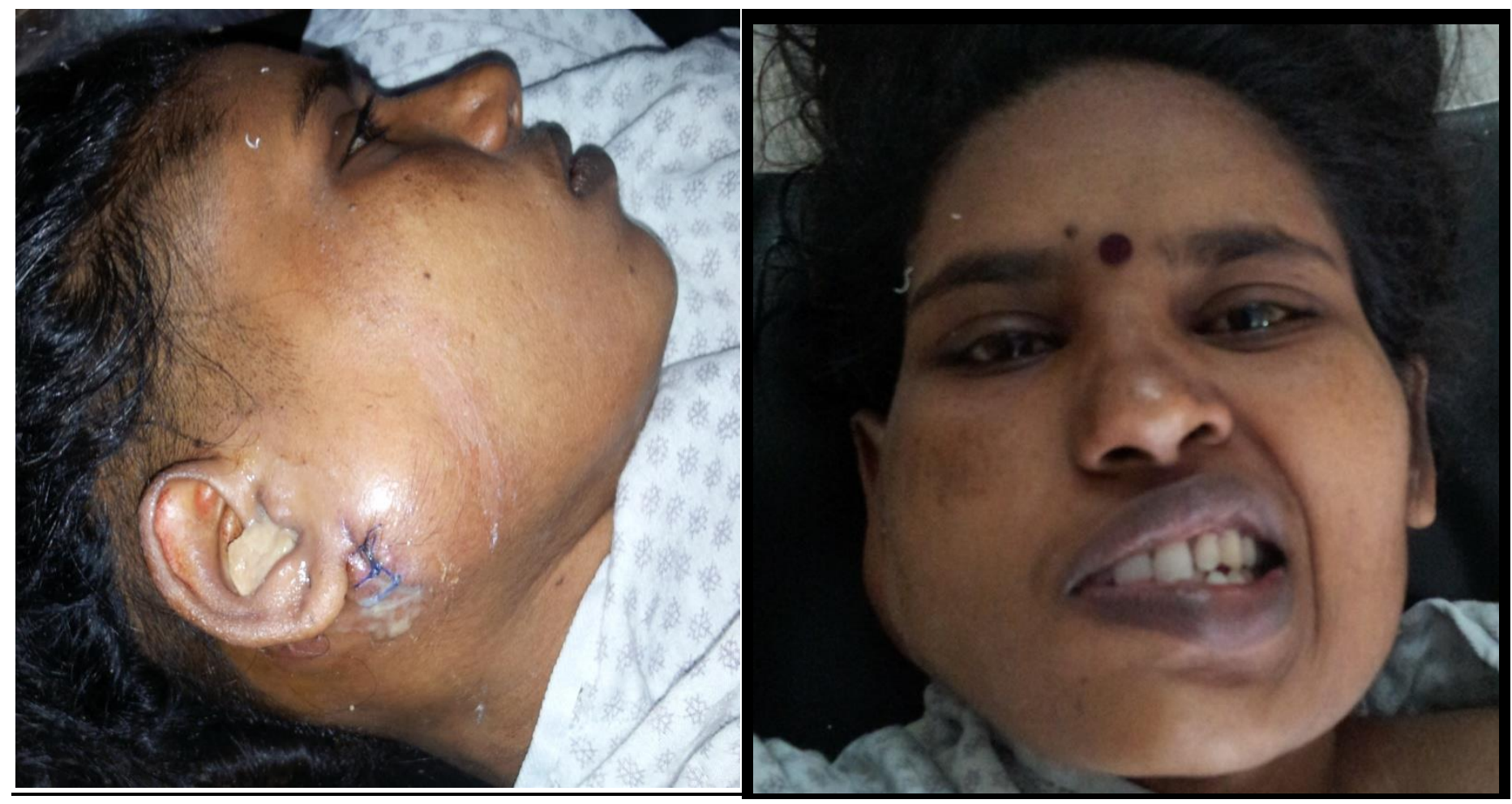

3. Swelling over Rt parotid and post aural region Since 2 months which was gradual in onset and progressive in nature associated with pain of severe type, throbbing in nature, not radiating.

4. After 4 days of swelling patient develops deviation of mouth and improper closure of right eye (Right facial palsy) which was sudden in onset. Patient also c/o sudden change in voice and difficulty in deglutition.

\section{Investigations}

1. FNAC from parotid swelling done on September 2013 and report came sialadenitis with reactive changes.

2. Incisional biopsy of right parotid swelling was done and report came FNAC-acute inflammatory lesion with epithelial atypia

3. HPE-completely necrosed tissue with fibrocollagenous tissue. Mixed inflammatory cell infiltrate.

MRI report- Lytic destructive lesion in petrous part of Right temporal bone, mastoid air cells, lateral margin of basiocciput, jugular tubercle, Right lateral margin of foramen magnum and squamous part / condylar portion of the right occipital bone, associated with large soft tissue component. Soft tissue appears heterogeneously hyper intense on T2/FLAIR sequences and hypo intense on T1. It extends to right external, middle and few inner ear structures. Middle 
ear ossicles and facial canal on right side is completely infiltrated by lesion it also extends to carotid canal, jugular foramen. Inferiorly it engulfs part of ICA, IJV with partial infiltration in medial pterygoid muscle, right parapharyngeal space and deep lobe of parotid gland. Thrombosis is noted in right sigmoid sinus and IJV. Few enlarged lymph nodes were noted in level 2.

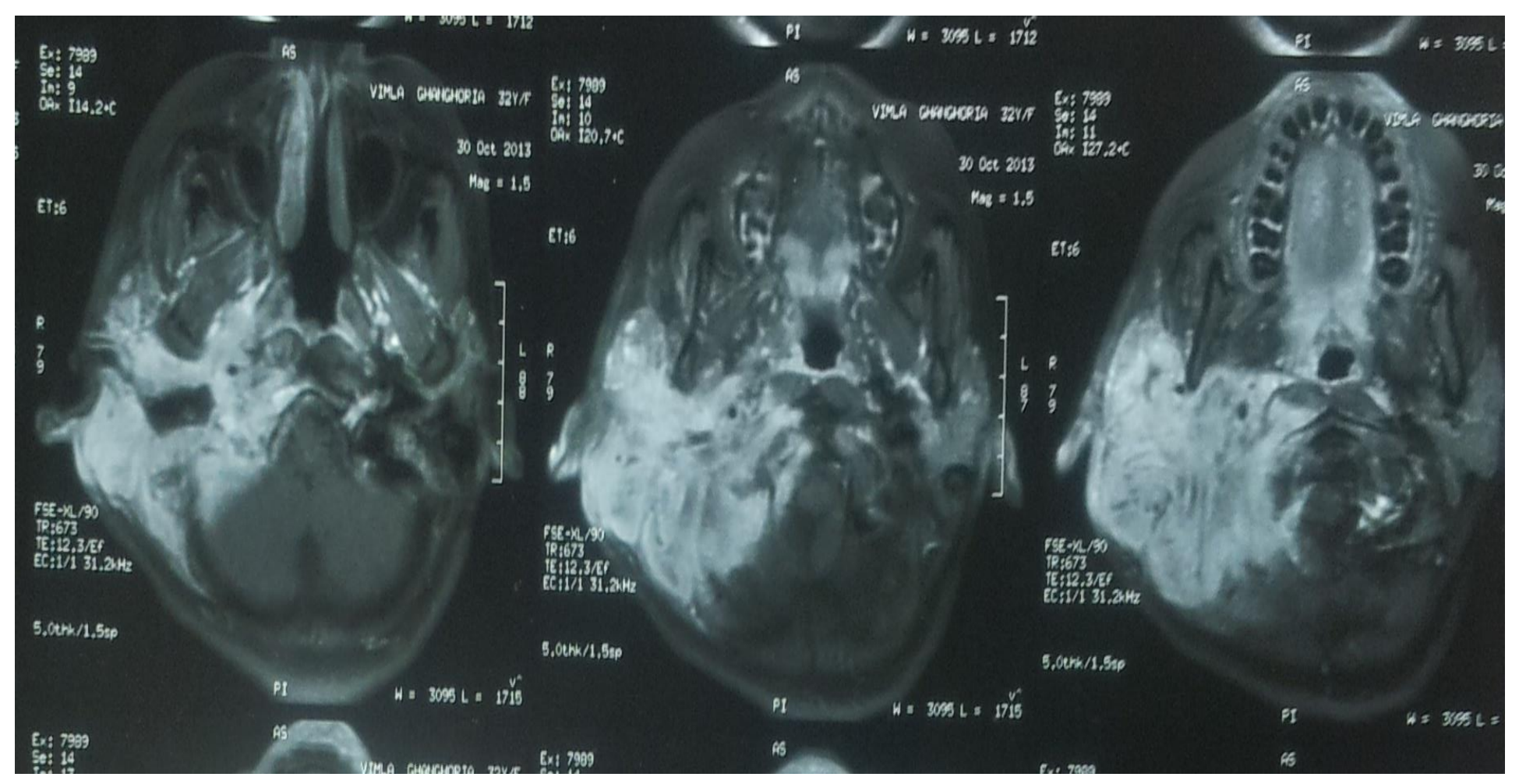

Impression -Large lytic lesion right lateral skull base involving petrous part of the rt temporal bone, mastoid air cells, lateral margins of the basiocciput, jugular tubercle, rt lateral margins of 
the foramen magnum \& squamous part/ condylar portion of the rt occipital bone, \&is associated with large soft tissue component.

\section{Management:}

With no definite preoperative diagnosis being made except MRI which was suggestive of malignancy, patient was taken up for surgery. She was operated at R D Gardi Medical College, Ujjain where a Mastoid exploration of right ear was done.

Per operatively we found a pale colored huge mass, rubbery in consistency along with destruction of mastoid antrum, attic, aditus was also present. Dural and sinus plates were eroded, jugular bulb was exposed, all the ossicles were absent. Mastoid segment of facial nerve was exposed \& granulations were present over it.

Excised tissue from mastoid and middle ear was sent for histopathological examination which revealed an Invasive Adenocarcinoma - middle ear right side.

Histopathology report in detail was: Gross- multiple grayish white soft tissue pieces of $0.1 \mathrm{gm}$. Microscopic - section shows nests and trabeculae of compact clusters of cuboidal cells with central monomorphic nuclei showing central nucleoli, cytoplasm was eosinophillic. Mitosis was seen infrequently. Tumor cell trabeculae show peripheral palisading. Foci of squamous metaplasia were seen. Tumor is infiltrating dense fibrous tissue.

Patient was subjected to post-operative radiotherapy.

\section{Discussion}

Middle ear adenomas are unusual neoplasms with epithelial and neuroendocrine differentiations.

Hyams and Michaels were the first to hypothesize that MEA originated from the mucosal epithelium of the middle ear, [1]

Gaffey et al in 1988 proposed the term "aggressive papillary middle ear tumour" for adenomatous middle ear tumours having papillary architecture[2,3]

These tumours were extremely aggressive locally invading bone and possibly extending intracranially

Paulus etal. 1999 [4] described a 53-year-old man who suffered from otalgia and tinnitus for more than 10 years and from neurological deficits for 1 year due to a large temporal bone tumor that invaded the temporal lobe. A combined neurosurgical and otolaryngological resection was performed. Pathological analysis revealed a low-grade adenocarcinoma of a mixed epithelial-neuroendocrine phenotype, which showed a close histological similarity to, and topographical relationship with, middle ear epithelium. The authors conclude that middle ear adenocarcinoma belongs to the spectrum of extracranial tumors that have possible local extension to the brain. 
Wynn Adam etal (5) suggested that Adenocarcinoma of the middle ear is a rare condition. It should be considered in the differential diagnosis of lytic lesions of the temporal bone particularly in a patient with a history of chronic ear infection. He also suggested that the radiographic manifestations are nonspecific and are common to other malignancies of the mastoid.

Marioni G etal (6) that both CT and MRI must be done in such cases. Computerized tomography is useful in detecting bone involvement beyond the external canal, giving a direct representation of the markedly destructive pathological process. Magnetic resonance has better soft tissue contrast, permitting more accurate evaluation of the cartilaginous portion of the external auditory canal

We reported a case of $24 \mathrm{yr}$ old female patient presenting as chronic Suppurative otitis media of long duration with parotid swelling and sudden onset of facial palsy MRI reveals extensive spread of middle ear tumour to intraspinal \& intracranial posterior fossa, sigmoid \& transverse venous sinuses with contiguous involvement of entire parotid substance. In this case mastoid exploration is done \& pathological analysis of biopsy tissue revealed invasive adenocarcinoma.

Recurrences is also a common feature of these tumors as reported in many case reports[7,8]. Complete surgical removal of the neoplasm depending on the extent of tumour with postoperative radiotherapy is recommended treatment for these tumors.

Adenomas and adenocarcinomas originating from the middle ear are few in number and have met with significant controversy in the literature. These glandular tumors engender controversy regarding histologic origin and the pathologic demarcation between adenoma and adenocarcinoma. However aggressive clinical behavior can be the result of lesions that appear microscopically benign. Furthermore, we present evidence of the similarity of these glandular lesions to paragangliomas and carcinoid tumors. [9]

Wynn Adam etal (5) also suggested that the differential diagnosis for a destructive lesion of the temporal bone which includes Primary neoplasms include squamous cell carcinoma, adenoid cystic carcinoma, adenocarcinoma, osteosarcoma, chondrosarcoma, rhabdomyosarcoma, paraganglionoma], hemangioma, facial nerve neuroma, meningioma, teratoma, giant cell tumor, and hemangiopericytoma. Secondary malignancies may produce bony changes by local invasion, by blood-borne metastases (particularly from breast, lung, kidney GIT, and Thyroid), and by involvement with hematologic malignancies such as multiple myeloma or leukemia

\section{Conclusion:}

Primary Middle ear adenocarcinoma is a very rare, locally invasive neoplasm assumed to arise from the middle ear mucosa. It should be considered in the differential diagnosis of lytic lesions of the temporal bone particularly in a patient with a history of chronic ear infection. High degree of suspicion is needed in patients of Chronic ear infections with a progressively increasing mass in the ar with or without facial palsy and early diagnosis and management is 
the key to a good long term results. A 5-year survival of $70 \%$ has been reported for low-grade adenocarcinomas.(10)

\section{References:}

1.Hyams VJ, Michaels L: Benign adenomatous neoplasm (adenoma) of the middle ear.

Clin Otolaryngol Allied Sci 1976, 1:17-26. pubmed

2) Gaffey MJ, Mills SE, Fechner RE, Intemann SR, Wick MR. Aggressive papillary middle-ear tumor. A clinicopathologic entity distinct from middle-ear adenoma. Am J Surg Pathol. 1988;12:790-7. doi: 10.1097/00000478-198810000-00009. [PubMed] [Cross Ref]

3) Polinsky MN, Brunberg JA, McKeever PE, sandler HM, Telian S, Ross D. Aggressive papillary middle ear tumors: a report of two cases with review of the literature. Neurosurgery. 1994;35:493-7. doi: 10.1227/00006123-19940900000018. [PubMed] [Cross Ref]

4) Paulus W, Romstock J, Weidenbecher M, Huk WJ, Fehlbusch R. Middle ear adenocarcinoma with intracranial extension, Case report. J of Neurosurgery. 1999;90(3):555-8. doi: 10.3171/jns.1999.90.3.0555. [PubMed] [Cross Ref]

5) Wynn Adam,1 John C. Johnson,1 David J. Paul,1 Kathryn Clausen,2 and David E. Schuller3 - Primary Adenocarcinoma of the Middle Ear - . AJNR 3:674-676, November / Oecember 1982

6) Marioni G. ${ }^{a}$ - de Filippis C. ${ }^{a} \cdot$ Stramare R. ${ }^{b} \cdot$ Puggina S. ${ }^{b} \cdot$ Marino F. ${ }^{c} \cdot$ Ferran-Bennström E. $^{a} \cdot$ Staffieri A - Intracranial Invasion from Primary Adenocarcinoma Arising in the External and Middle Ear- Otorhinolaryngol Nova 2001;11:308-313

7) Diagn Pathol. 2011; 6: 62. Published online Jul 7, 2011. doi: 10.1186/1746-1596-662Aggressive low grade middle ear adenocarcinoma with multiple recurrences: a case report Nadia G Elhefnawy

8) D E Schuller, J J Conley, J H Goodman, K P Clausen, W J Miller - Primary adenocarcinoma of the middle ear. Otolaryngology Head and Neck Surgery (Impact Factor: 1.72). 07/1983; 91(3):280-3. Source: PubMed

9) Amble FR, Harner SG, Weiland LH, Mc Donald TJ, Facer GW. Middle ear adenoma and adenocarcinoma. Otolaryngol Head neck Surg. 1993;109:871-6. [PubMed]

10) Burhan Dadaș · Seyhan Alkan · Suat Turgut Tülay Bașak - Primary papillary adenocarcinoma confined to the middle ear and mastoid - - Eur Arch Otorhinolaryngol (2001) $258: 93-95$ 